\title{
Considerations on the 'harmonic balancing approach to nonlinear oscillations of a punctual charge in the electric field of charged ring'
}

A. Beléndez ${ }^{1,2}$, E. Fernández ${ }^{3}$, J.J. Rodes ${ }^{1}$, R. Fuentes ${ }^{2,3}$ and I. Pascual ${ }^{2,3}$

${ }^{1}$ Departamento de Física, Ingeniería de Sistemas y Teoría de la Señal. Universidad de Alicante. Apartado 99. E-03080 Alicante. SPAIN

${ }^{2}$ Instituto Universitario de Física Aplicada a las Ciencias y las Tecnologías.

Universidad de Alicante. Apartado 99. E-03080 Alicante. SPAIN

${ }^{3}$ Departamento de Óptica, Farmacología y Anatomía.

Universidad de Alicante. Apartado 99. E-03080 Alicante. SPAIN

E-mail: a.belendez@ua.es

Corresponding author: A. Beléndez

Phone: +34-96-5903651

Fax: +34-96-5909750 


\begin{abstract}
In a previous short communication [Physics Letters A 373 (2009) 735-740] the nonlinear oscillations of a punctual charge in the electric field of a charged ring were analyzed. Approximate frequency-amplitude relations and periodic solutions were obtained using the harmonic balance method. Now we clarify an important aspect about of this oscillation charge. Taking into account Earnshaw's theorem, this punctual charge cannot be a free charge and so it must be confined, for example, on a finite conducting wire placed along the axis of the ring. Then, the oscillatory system may consist of a punctual charge on a conducting wire placed along the axis of the uniformly charged ring.
\end{abstract}

Keywords: Nonlinear oscillator; equilibrium point; Earnshaw's theorem. 
In a previous paper [1] we obtain approximate periodic solutions to the oscillations of a charge in the electric field of a charged ring by applying the harmonic balance method. To do this, the following dimensionless second-order nonlinear differential equation was solved approximately

$$
\frac{\mathrm{d}^{2} y}{\mathrm{~d} \tau^{2}}+\frac{y}{\left(1+y^{2}\right)^{3 / 2}}=0
$$

with initial conditions

$$
y(0)=A \quad \text { and } \quad \frac{\mathrm{d} y}{\mathrm{~d} \tau}(0)=0
$$

In order to obtain Eq. (1) we considered a uniformly charged ring of radius $R$ with a charge $Q>0$, whose electric field $E$ on the axis (say the $x$-axis) of the ring was given by

$$
E(x)=\frac{1}{4 \pi \varepsilon_{0}} \frac{Q x}{\left(R^{2}+x^{2}\right)^{3 / 2}}
$$

and a negative punctual charge $q=-|q|$ placed at an arbitrary on-axis point. We did not actually specify that this punctual charge was a free charge, but this could be concluded from the context. The stable equilibrium position is located in the centre of the ring and the punctual charge oscillates between symmetric bounds $[+A,-A]$. However, an important aspect of this physical system is that the punctual charge $q$ cannot be a free charge because Earnshaw's theorem states that no stable equilibrium point may be found for a free charge in space in the presence of an electric field generated by a charge distribution [2-4]. Therefore, there are no local minima or maxima of the field potential in free space, only saddle points. Hence, the centre of the ring is not a stable equilibrium point and oscillations of the charge around this point are not possible. To solve this problem it could be possible to consider that the punctual charge is on a finite conducting wire placed along the axis of the ring as it is done as in reference 4 . The charge must be confined within the wire, since the charge has to oscillate on the ring axis, in the proximity of its stable equilibrium point located in the centre of the ring [3]. 
In any case, in accordance with Earnshaw's theorem, confinement of the charge along the axis of the ring is a necessary requirement. Under this condition, nonlinear oscillations of the punctual charge along the ring axis in the proximity of its centre are possible.

\section{Acknowledgements}

This work has been supported by the "Generalitat Valenciana" of Spain (projects ACOMP/2009/150 and ACOMP/2009/160).

\section{References}

[1] A. Beléndez, E. Fernández, J. J. Rodes, R. Fuentes and I. Pascual, "Harmonic balancing approach to nonlinear oscillations of a punctual charge in the electric field of charged ring”, Phys. Lett. A 373, 735-740 (2009).

[2] S. Earnshaw, "On the nature of the molecular forces which regulate the constitution of the luminiferous ether", Trans. Camb. Phil. Soc. 7, 97-112 (1842).

[3] J. D. Jackson, Classical Electrodymamics (Wiley, New York, 1975).

[4] R. De Luca and S. Ganci, "Classical charge oscillations in nanoscopic systems", Rev. Bras. Ens. Fis. 31, 2310 (2009). 